\title{
Integration of CTA in the Diagnostic Workup of New Onset Chest Pain in Clinical Practice
}

\author{
Nazario Carrabba $\mathbb{D}^{1},{ }^{1}$ Martina Berteotti, ${ }^{1}$ Giulia Taborchi, ${ }^{1}$ \\ Francesca Ciatti, ${ }^{1}$ Manlio Acquafresca, ${ }^{2}$ Mario Moroni, ${ }^{2}$ Angela Migliorini, ${ }^{1}$ \\ Vittorio Miele $\mathbb{I D}^{2}{ }^{2}$ Niccolò Marchionni, ${ }^{1}$ and Renato Valenti ${ }^{1}$ \\ ${ }^{1}$ Cardiovascular and Thoracic Department, Careggi Hospital, Florence, Italy \\ ${ }^{2}$ Radiology Department, Careggi Hospital, Florence, Italy
}

Correspondence should be addressed to Nazario Carrabba; n.carrabba@virgilio.it

Received 28 March 2019; Accepted 16 June 2019; Published 7 July 2019

Academic Editor: Nicola Gaibazzi

Copyright (C) 2019 Nazario Carrabba et al. This is an open access article distributed under the Creative Commons Attribution License, which permits unrestricted use, distribution, and reproduction in any medium, provided the original work is properly cited.

\begin{abstract}
Background. Recently, NICE guidelines recommend the use of computed tomographic angiography (CTA) as the first line of investigation for new onset chest pain. We sought to evaluate the impact of the integration of CTA in the diagnostic workup, as either a first- or second-line of investigation, in the clinical practice for patients presenting with new onset chest pain, with suspicion that it may be due to coronary artery disease (CAD). Method and Results. From 2014 to 2016, 208 outpatients (mean age $63.8 \pm 12.7,37 \%$ female) with an unknown CAD diagnosis were evaluated. About half $(\mathrm{n}=106,51 \%)$ received usual testing care plus CTA as a second-line investigation (group A), while the other half $(n=102,49 \%)$ received CTA as a first-line investigation (group $\mathrm{B})$. Care decisions and test interpretations were made by the attending physician. Obstructive CAD (O-CAD) was defined as $>50 \%$ stenosis in the principal branch. As determined by CTA, the rates of CAD in group A vs. group B were the following ( $\mathrm{P}=0.001$ ): $31.1 \%$ vs. $27.4 \%$ for normal/minimal CAD; $42.5 \%$ vs. $63.7 \%$ for no O-CAD; and $26.4 \%$ vs. $8.8 \%$ with O-CAD. Based on a diagnostic result of no O-CAD, invasive angiography was cancelled in $42.6 \%(n=45)$ of group A patients, and additional functional tests were cancelled for the same reason in $63.7 \%(n=65)$ of group B patients, without adverse events at median 3-year. The average diagnostic cost for patients in our study was lower in group B (206 vs. 324.42 euro; $\mathrm{P}<0.0001)$. Conclusions. In clinical practice, CTA, as a first- or second-line investigation, most commonly detected no O-CAD in new onset chest pain patients, leading us to safely avoid unnecessary ICA or additional functional tests. The use of CTA as a first-line investigation also appears to be cost saving, but its cost-effectiveness remains to be demonstrated in larger studies.
\end{abstract}

\section{Introduction}

For patients with suspected coronary artery disease (CAD), in many centers, the routine noninvasive diagnostic approach consists of stress testing, with the applied test modality depending on pretest probability, patient characteristics, local availability, and expertise. The exercise ECG is the most widely available and least expensive functional test, but it has a modest diagnostic performance. Conversely, the negative predictive value and the ability to exclude CAD are high for computed tomographic angiography (CTA) $[1,2]$. However, CTA has a tendency to overestimate both the angiographic and hemodynamic severity of CAD, producing results which may necessitate further functional testing or ICA. Because the prevalence of obstructive-CAD (O-CAD) is low in realworld populations with stable chest pain symptoms [3], CTA could be an efficient and cost-effective first-line test. Recently, the PROMISE [4] and SCOT-HEART [5] studies have suggested that an evaluation strategy based on coronary CTA improves diagnostic certainty, as well as the efficiency of triage to invasive catheterization. It also may reduce radiation exposure when compared with functional stress testing, with similar rates of cardiac events. Moreover, the EVINCI [6] trial supports the use of CTA for stable chest pain, highlighting better performance in comparison with other imaging strategies. Following the publication of these studies, 
the National Institute for Health and Care Excellence (NICE) [7] recommended CTA as the first-line investigation for all patients presenting with chest pain due to suspected CAD. In the present prospective registry, we sought to evaluate the impact of the integration of CTA as a first- or second-line investigation in the diagnostic workup for the evaluation of outpatients presenting with stable chest pain in our tertiary center.

\section{Material and Methods}

From May 2014 to February 2016, 334 consecutive symptomatic outpatients of age $\geq 18$ years old, with new onset chest pain, were referred to our Cardiovascular and Thoracic Department of Careggi Hospital (Florence, Italy) for the suspicion of CAD. Exclusion criteria for the prospective registry were previously documented CAD $(n=113)$, acute coronary syndrome or clinical instability $(n=5)$, contraindications to CTA $(n=2)$, and the need for emergent or urgent procedure $(n=1)$. Additional exclusion criteria included recent cardiovascular testing ( $<90$ days; $n=5$ ). Thus, 208 outpatients presenting with a low to intermediate likelihood of obstructive CAD were enrolled in this registry to evaluate the impact of integration of CTA in the diagnostic workup, using CTA as either a first- or second-line of investigation. Patients were enrolled by the attending physician, who, according to his behavior-and without any restriction-chose the patient's diagnostic workup, generating two groups of patients. Specifically, one group of patients receiving usual care testing (a symptom-limited exercise test, an echo stress test, or SPECT) according to the standard diagnostic workup, plus CTA as a second-line investigation, irrespective of the functional testing results, was classified as group A. Instead, patients receiving CTA as a first-line investigation were classified as group B (Figure 1). Care decisions and test interpretations were made by the attending physician, without any conditioning. All patients underwent CTA with a dual-source CTA scanner (52\% with high-pitch mode, depending on the baseline heart rate), and followed scanning protocols satisfying Society of Cardiac Computed Tomography quality standards [8]. Normal/minimal CAD was defined as $<30 \%$ stenosis in principal branches of the left or right coronary artery by visual estimate, while no O-CAD and O-CAD were defined as $\geq 30-50 \%$ and $>50 \%$ stenosis, respectively. All subjects provided informed written consent, which was approved by the local ethics committee. At least 3 authors (N.C., M.B., and G.T.) had access to all data presented. All authors are responsible for the data integrity. Followup information for the occurrence of death, myocardial infarction, or hospitalization for coronary angioplasty was obtained by clinical visits or telephone interviews over the course of the following 3 years. Hospital records of all patients were screened for the occurrence of clinical events to confirm the obtained information.

2.1. CTA Scan Protocol. All CTAs were performed using a 128-slice dual-source CTA system (SOMATOM Definition Flash, Siemens Healthineers, Forchheim, Germany). The detector collimation was $2 \times 64 \times 0.6 \mathrm{~mm}$, and a flying focal spot technique and a gantry rotation time of $280 \mathrm{msec}$ were used. Both tubes were operated at 100 or $120 \mathrm{kV}$, depending on the body mass index of the patient. Scout-based automatic tube current modulation (Care Dose 4D, Siemens healthcare, Forchheim, Germany) was used, with the reference tube current-time product set at $320 \mathrm{mAs}$ per rotation. The pitch was 3.4 for flash CTA mode. Oral and/or intravenous betablockers or oral ivabradine were administered if necessary, in an attempt to achieve a target heart rate $<60$ beats $/ \mathrm{min}$. All patients received sublingual nitrates. The contrast medium (Iomeron 400, Bracco Altana Pharma, Konstanz, Germany) and saline chaser were administered at $5 \mathrm{~mL} / \mathrm{s}$ using a dualhead power injector (Empower, ACIST) into an antecubital vein, through an 18-gauge catheter. The patient's heart rate and ECG trace were recorded during examination. A test bolus scan was performed to determine the transit time. An injection of $15 \mathrm{~mL}$ of iodinated contrast medium was followed by a $30 \mathrm{~mL}$ saline chaser. The time until the peak opacification in the proximal ascending aorta was measured, and this time, plus 2 for standard protocol, or plus 5 for high-pitch protocol, was considered to represent the transit time of contrast agent. Sixty-five milliliters of contrast medium, followed by a $50 \mathrm{~mL}$ saline chaser, was administered, with bolus tracking using a region of interest (ROI) in the ascending aorta. The scan was automatically triggered when the tracking ROI reached a threshold of 100 Hounsfield units (HU) above baseline attenuation. In the flash mode (high-pitch spiral mode), prospective ECG-triggering was used to obtain a complete dataset in a single heartbeat starting at $60 \%$ of the R-R interval. In the sequential mode (Spiral technique), the centre of the data acquisition window was set at $70 \%$ of the R-R interval. The entire heart was covered in three or four heartbeats in a step-and-shoot fashion [9].

2.2. Image Reconstruction and Evaluation. Datasets for coronary arteries were reconstructed with a slice thickness of 0.6 $\mathrm{mm}$, an increment of $0.4 \mathrm{~mm}$, a field of view of $180 \mathrm{~mm}$, a medium-soft convolution kernel (B26), and, in patients exhibiting coronary calcium, an additional sharp convolution kernel (B46). All reconstructed images were transferred to a dedicated workstation (MMWP, Siemens Healthcare, Forchheim, Germany). Axial images, multiplanar reformations, and maximum intensity projections were used to evaluate arteries. Coronary artery segments were classified according to a modified American Heart Association [10] protocol. Segments were evaluated if the luminal diameter met or exceeded $1.5 \mathrm{~mm}$, as judged by two independent observers (N.C. and M.A., each with more than 9 years of coronary CTA experience). Any discordance in the interpretation was solved by a third observer (M.M. with more than 7 years reading experience). Image quality was assessed semiquantitatively using a four-point grading scale: (1) excellent (no artifacts, unrestricted evaluation), (2) good (minor artifacts, good diagnostic quality), (3) adequate (moderate artifacts, still acceptable and diagnostic), and (4) not assessable (severe artifacts impairing accurate evaluation). Images with a score of 1-3 were considered acceptable for diagnosis [8]. 


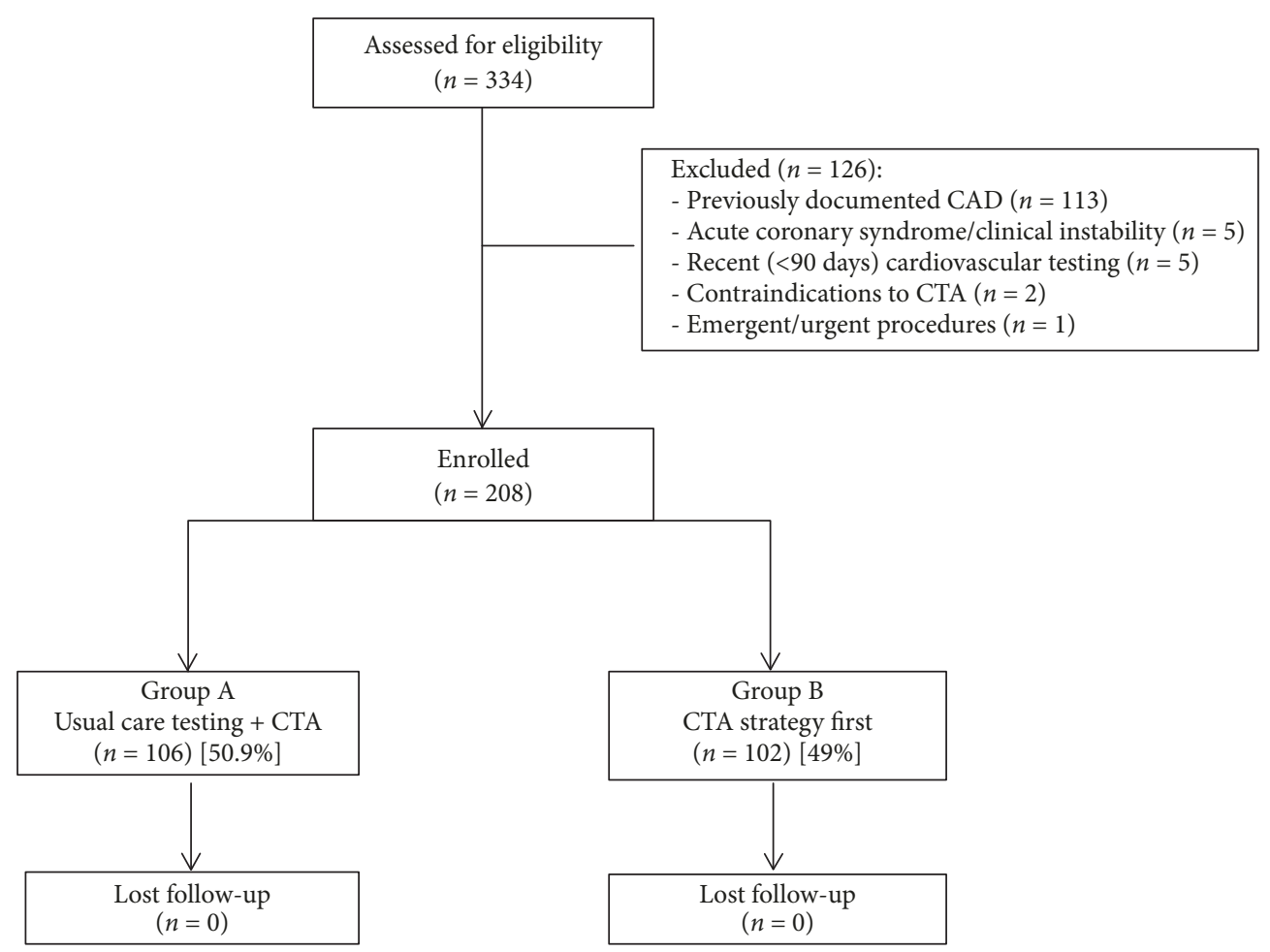

FIGURE 1: Study design flow diagram. CAD: coronary artery disease; CTA: computed tomographic angiography.

2.3. Radiation Dose Estimates. The radiation dose was reported as dose-length product (DLP) and effective dose (ED). For each patient, the ED was calculated using the formula DLP x 0.014, using the 0.014 conversion factor for chest radiation (in $\mathrm{mSv} / \mathrm{Gy} / \mathrm{cm}$ ) [11].

2.4. Statistical Analysis. All variables are expressed as mean value \pm SD or medians with interquartile ranges (25th to 75 th percentile). Differences in patient characteristics, radiation dose, and image quality were compared using an independent-sample $t$-test (if normally distributed) or Mann-Whitney U-test (if not) for continuous variables, using $\chi^{2}$ or Fisher's exact test for categorical variables and the Kruskal-Wallis test for nonparametric data, as appropriate. The interrater agreement between the two observers in assessing image quality was calculated using Cohen's kappa statistics. Kappa results were interpreted as being either poor $(\kappa<0.20)$, fair $(\kappa=0.21-0.40)$, moderate $(\kappa=0.41-0.60)$, good $(\kappa=0.61-0.80)$, very good $(\kappa=0.81-0.90)$, or excellent $(\kappa \geq 0.91)$. For economic analysis, unadjusted diagnostic costs were compared between the diagnostic strategies deployed by groups A and B using the nonparametric Wilcoxon rank sum test on all patients. For this economic analysis, the cost for the exercise test is 113.5 euros, the echo stress test is 181 euros, SPECT is 433 euros, and the CT scan is 206 euros. Statistical analyses were performed using commercially available software (SPSS, version 19.0, Chicago, IL, USA). A twosided p-value of less than 0.05 was considered statistically significant.

\section{Results}

The 208 patients in the registry averaged $63.8 \pm 12.7$ years of age, and $37 \%$ were women. The clinical characteristics of the patients were similar, but group B $(n=102,49 \%)$ was older and the rate of female gender was higher in comparison with group A $(n=106,51 \%)$. All patients showed an intermediate risk profile (see Table 1). Among the patients in group A, 102 (96\%) underwent symptom-limited exercise electrocardiography with the standard Bruce protocol, 3 (3\%) underwent an echo stress test, and $1(1 \%)$ was tested by SPECT. According to the results of the usual care strategy, group A patients were classified as exhibiting a negative $(n=54,50.9 \%)$, inconclusive $(n=44,41.5 \%)$, or positive $(n=8$, $7.5 \%$ ) result of the functional test. Based on the results of CTA, as per patient analysis, the overall rate of patients with no O-CAD (52.8\%) was the highest when compared to the rates of minimal/normal coronary artery (29.3\%) and O-CAD (17.7\%). Specifically, in group A, the percentage of normal/minimal lesion of coronary artery was $31.1 \%$ vs. $27.4 \%$ in group $\mathrm{B}$; the percentage of no significant $\mathrm{O}-\mathrm{CAD}$ was $42.5 \%$ in group A vs. $63.7 \%$ in group B; and the percentage of $\mathrm{O}-\mathrm{CAD}$ was $26.4 \%$ in group $\mathrm{A}$ vs. $8.8 \%$ in group $\mathrm{B}(\mathrm{P}=0.001)$ (Table 2). Per vessel analysis, the number of diseased coronary vessels was not significantly different in group A vs. group B patients (Table 2). Moreover, in patients with coronary risk factor $(\mathrm{RF}) \geq 2$ compared with patients with a coronary $\mathrm{RF}$ of $0-1$, the number of diseased coronary vessels $(\mathrm{P}=0.0035)$ (Figure 2A), as well as the number of patients with proximal plaques ( $\mathrm{P}=0.0009)$ (Figure $3 \mathrm{~A})$, was higher. Additionally, the 
TABLE 1: Baseline clinical characteristics of the study population.

\begin{tabular}{|c|c|c|c|c|}
\hline & $\begin{array}{c}\text { All } \\
(\mathrm{n}=208)\end{array}$ & $\begin{array}{l}\text { Group A } \\
(n=106)\end{array}$ & $\begin{array}{l}\text { Group B } \\
(\mathrm{n}=102)\end{array}$ & $\mathrm{p}$-value \\
\hline Mean age (years) & $63.8 \pm 12.7$ & $61.5 \pm 12.5$ & $66.17 \pm 12.7$ & 0.008 \\
\hline Male gender, n (\%) & $131(63 \%)$ & $77(72.7 \%)$ & $54(52.9 \%)$ & 0.004 \\
\hline Obesity, n (\%) & $8(3.8 \%)$ & $3(2.8 \%)$ & $5(4.9 \%)$ & 0.492 \\
\hline Hypertension, n (\%) & $114(54.8 \%)$ & $54(50.9 \%)$ & $60(58.9 \%)$ & 0.267 \\
\hline Hypercholesterolemia, n (\%) & $67(32.2 \%)$ & $39(36.8 \%)$ & $28(27.4 \%)$ & 0.181 \\
\hline Diabetes mellitus, n (\%) & $33(15.9 \%)$ & $21(19.8 \%)$ & $12(11.8 \%)$ & 0.130 \\
\hline Smoking habits, n (\%) & $45(21.6 \%)$ & $18(17.0 \%)$ & $27(26.4 \%)$ & 0.129 \\
\hline Family history of CAD, n (\%) & $57(27.4 \%)$ & $31(29.2 \%)$ & $26(25.5 \%)$ & 0.641 \\
\hline Pretest probability & $55.97 \pm 30.92$ & $55.33 \pm 22.05$ & $56.73 \pm 21.42$ & 0.692 \\
\hline
\end{tabular}

Data are presented as the mean \pm SD or as number (percentage) of patients; CAD: coronary artery disease; hypercholesterolemia: total cholesterol $>5 \mathrm{mmol} / \mathrm{L}$, low-density lipoprotein $>3 \mathrm{mmol} / \mathrm{L}$, or on lipid-lowering medication.

TABLE 2: CTA angiographic characteristics by groups.

\begin{tabular}{|c|c|c|c|}
\hline & $\begin{array}{l}\text { Group A } \\
(\mathrm{n}=106)\end{array}$ & $\begin{array}{l}\text { Group B } \\
(\mathrm{n}=102)\end{array}$ & p-value \\
\hline \multicolumn{4}{|l|}{ Analysis by patient } \\
\hline No lesions or minimal lesions, $\mathrm{n}(\%)$ & $33(31.1)$ & $28(27.4)$ & 0.001 \\
\hline No obstructive lesions, $\mathrm{n}(\%)$ & $45(42.6)$ & $65(63.7)$ & \\
\hline Obstructive lesions, $\mathrm{n}(\%)$ & $28(26.4)$ & $9(8.8)$ & \\
\hline 1 vessel, $\mathrm{n}(\%)$ & $10(9.4)$ & $18(17.6)$ & 0.369 \\
\hline 2 vessels, $\mathrm{n}(\%)$ & $15(14.1)$ & $14(13.7)$ & \\
\hline 3 vessels, n (\%) & $11(10.4)$ & $8(7.8)$ & \\
\hline Left main, $\mathrm{n}(\%)$ & $38(35.8)$ & $34(33.3)$ & \\
\hline \multicolumn{4}{|l|}{ Analysis by segment } \\
\hline No lesions or minimal lesions, median (IQR) & $0(0-0)$ & $0(0-0)$ & 0.164 \\
\hline Non obstructive lesions, median (IQR) & $0(0-3)$ & $2(0-4)$ & 0.003 \\
\hline Obstructive lesions, median (IQR) & $0(0-1)$ & $0(0-0)$ & 0.001 \\
\hline \multicolumn{4}{|l|}{ Analysis by plaque } \\
\hline Noncalcified plaques, median (IQR) & $0(0-0)$ & $0(0-0)$ & 0.015 \\
\hline Partially calcified plaques, median (IQR) & $0(0-3)$ & $1(0-2)$ & 0.870 \\
\hline Calcified plaques, median (IQR) & $0(0-1)$ & $0(0-1)$ & 0.296 \\
\hline
\end{tabular}

Data are presented as number (\%) or median (IQR: interquartile range).

number of diseased coronary vessels $(\mathrm{P}=0.0199)$ (Figure $2 \mathrm{~B})$, as well as the number of patients with proximal plaques $(\mathrm{P}=0.0173)$ (Figure 3B), was higher in patients with an intermediate or high tertile risk profile in comparison to patients with a low tertile risk. Per segment analysis, in group B patients, when compared with group A patients, the number of segments showing no obstructive plaques was higher $(\mathrm{P}=0.0034)$, whereas the number of segments showing obstructive plaques was lower, and the number of segments with no or minimal lesion was not different between the two groups (Table 2). Per plaque analysis, the noncalcified plaques were more frequently observed in group $B$ patients with respect to group A patients, without difference in calcified or partially calcified plaques (Table 2). In group A patients, CTA, respectively, demonstrated 13 (24\%), 10 (22.7\%), and $5(62.5 \%)$ patients with O-CAD, among patients whose results showed negative, inconclusive, and positive functional tests (see Table 3). After examining the results of CTA, the planned investigations changed mainly due to the exclusion or identification of O-CAD. Specifically, among group A patients, the $42.5 \%$ identified with no O-CAD had ICA cancelled, and $31.1 \%$ with normal/minimal CAD did not undergo other tests. Among group B patients, the $63.7 \%$ with no O-CAD avoided functional testing, and the 27.4\% with normal/minimal CAD did not undergo other tests. The $26.4 \%$ and the $8.8 \%$ of patients with O-CAD among groups $\mathrm{A}$ and $\mathrm{B}$, respectively, underwent ICA directly without additional noninvasive functional tests, considering the availability in catheterization lab of IVUS and FFR in case of the persistence of uncertainty of the significance of coronary stenosis after ICA evaluation. Overall, in patients presenting with O-CAD by CTA $(n=37)$, ICA confirmed obstructive CAD in $92 \%(23 / 25)$ of cases. In 2 patients, the intravascular ultrasound did not confirm a case of O-CAD detected by CTA, demonstrating an overestimation of the degree of the coronary stenosis (sensitivity 100\%, specificity 


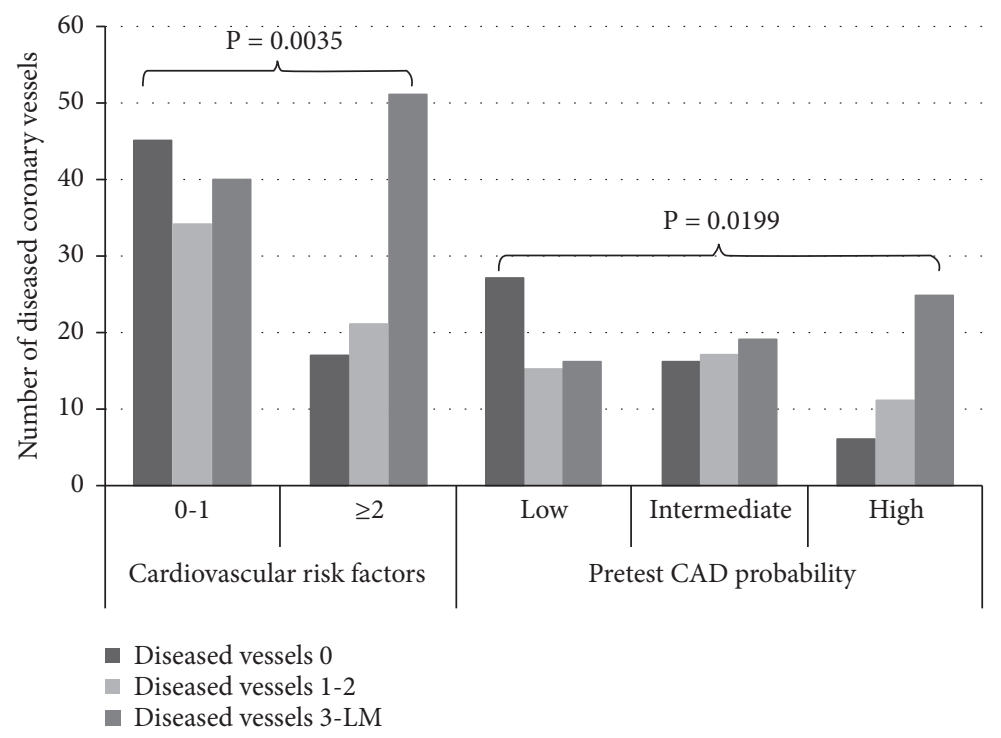

FIGURE 2: A: relationship between number of diseased coronary vessels and coronary risk factor $(\mathrm{RF}) \geq 2$ vs. $\mathrm{RF} 0-1$ ( $\mathrm{P}=0.0035)$. B: relationship between number of diseased coronary vessels and pretest probability low vs. intermediate and high $(\mathrm{P}=0.0199)$.

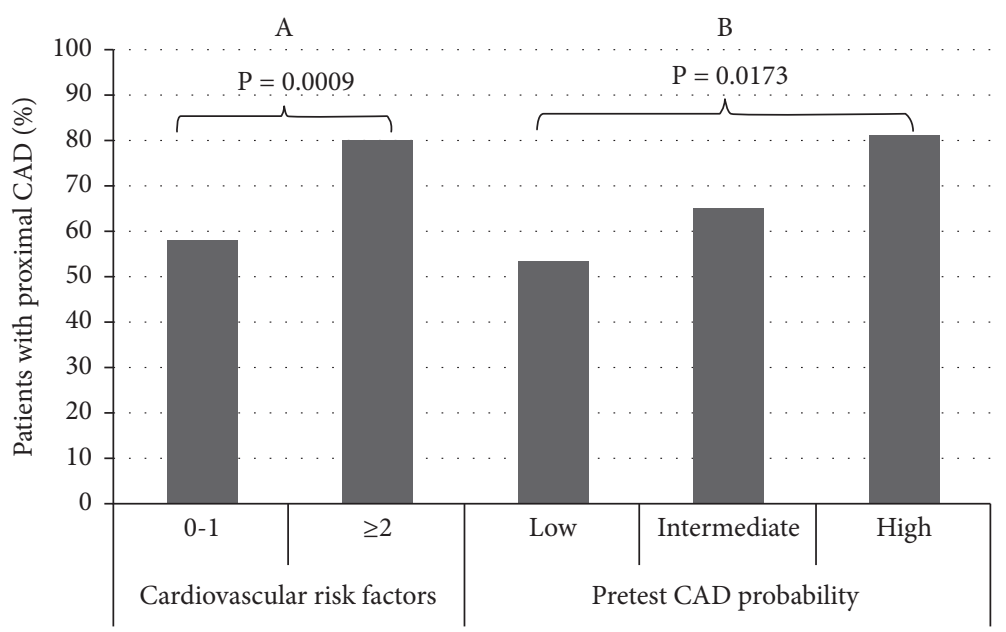

FIGURE 3: A: relationship between percentage of patients with proximal plaques and coronary risk factor ( $R F) \geq 2$ vs. $R F(0-1$ ( $P=0.0009)$. $B$ : relationship between percentage of patients with proximal plaques and pretest probability low vs. intermediate and high $(\mathrm{P}=0.0173)$.

$60 \%$, positive predictive value $92 \%$ [ $95 \%$ CI 75.3 to 97.77 ], negative predictive value $100 \%$ [ $95 \%$ CI 43.85 to $99.99 \%$ ], and accuracy $92.85 \%$ ). In the remaining 12 patients, ICA was not performed due to plaques located in distal part of coronary vessel in 5 patients ( 3 from group A) and in minor branches in 7 patients (3 from group A). These patients with stable OCAD were treated with optimal medical therapy. In case of the persistence of symptoms despite medical treatment, a PCI was performed 3 months after CTA, after the demonstration of large amount of myocardial ischemia $(n=3)$ or positive fractional flow reserve $(n=1)$.

3.1. Cost Analysis. The cost analysis revealed that the average diagnostic cost per patient for patients in group A was 324.42 euros (95\% CI: 310.34-338.51); for patients in group B, it was 206 euros $(\mathrm{P}<0.0001)$. Thus, the diagnostic cost-savings was a difference of 118.42 euros while using CTA as a first-line investigative strategy, as compared to the standard strategy.

3.2. High-Pitch vs. Standard CTA. The patient heart rate was lower in those who underwent high-pitch CTA in comparison to those who underwent standard CTA $(55 \pm 4$ bpm vs. $67 \pm 7 \mathrm{bpm}, \mathrm{P}<0.001)$. The mean cumulative radiation exposure was also lower in patients receiving high-pitch vs. standard CTA mode $(1.48 \pm 1.36 \mathrm{mSv}$ vs. $8.10 \pm 3.14 \mathrm{mSv}$, $\mathrm{P}<0.001)$. The agreement between the two observers in assessing image quality was excellent, with a kappa result of 0.93 . The number of segments exhibiting coronary vessels not 
TABLE 3: Functional test and CTA results of Group A and Group B patients.

\begin{tabular}{|c|c|c|c|c|}
\hline \multirow[t]{2}{*}{ Functional test results } & & \multicolumn{3}{|c|}{ CTA results } \\
\hline & & Obstructive CAD & No obstructive CAD & Minimal lesions/no CAD \\
\hline \multicolumn{5}{|l|}{ Group A $(n=106)$} \\
\hline All, n (\%) & & $28(26.4 \%)$ & $45(42.5 \%)$ & $33(31.1 \%)$ \\
\hline Negative, n (\%) & $54(50.9 \%)$ & $13(24.1 \%)$ & $23(42.6 \%)$ & $18(33.3 \%)$ \\
\hline Positive, n (\%) & $8(7.5 \%)$ & $5(62.5 \%)$ & $1(12.5 \%)$ & $2(25.0 \%)$ \\
\hline Inconclusive, n (\%) & $44(41.5 \%)$ & $10(22.7 \%)$ & $21(47.7 \%)$ & $13(29.5 \%)$ \\
\hline \multicolumn{5}{|l|}{ Group B $(n=102)$} \\
\hline All, n (\%) & & $9(8.8 \%)$ & $65(63.7 \%)$ & $28(27.4 \%)$ \\
\hline Negative, n (\%) & - & - & - & - \\
\hline Positive, n (\%) & - & - & - & - \\
\hline Inconclusive, n (\%) & - & - & - & - \\
\hline
\end{tabular}

Data are presented as number (percentage).

able to be evaluated (quality score $=4$ ) was low and similar in both high-pitch and standard CTA mode: $1.2 \%$ vs. $1.04 \%$ $(\mathrm{P}=0.634)$, respectively. Following CTA, an allergic reaction to the contrast medium agent occurred in 1 patient, who was successfully treated with cortisone and antihistamine agents.

3.3. Follow-Up. Throughout a median 3-year follow-up (group A 36.3 \pm 11.2 months and group B 36.7 \pm 11.4 months, $\mathrm{P}=0.798$ ), death and myocardial infarction did not occur in any of the patients with no O-CAD and cancelled ICA (from group A) nor in those with a cancelled functional test (from group B). The unplanned evaluations in the emergency department included two cases of palpitations, one for each group, and two cases of acute chest pain, one for each group; both were discharged from the hospital uneventfully.

\section{Discussion}

Currently, a great debate occurs about the functional and anatomic diagnostic evaluation of CAD in patients presenting with new onset chest pain. After the publication of recent studies [4-6], the NICE guidelines recommended the use of coronary CTA as a first-line diagnostic test evaluation for all patients presenting with new onset chest pain suspected for CAD [7]. Our findings confirm that in clinical practice the use of coronary CTA, given its strong negative predictive value [12], allows us to avoid unnecessary functional tests or a more expensive and potentially harmful ICA in patients showing normal coronary arteries or no O-CAD. Undoubtedly, CTA increased the identification of both obstructive and no O-CAD. While currently there is no clear guidance on how to manage the case of no O-CAD, the most common pattern of atherosclerosis observed in new onset chest pain patients, there is growing evidence of the beneficial effects of statin therapy [13]. Not surprisingly, in our registry, the burden of diseased vessels and the rate of proximal plaques by CTA increased with the presence of more than two coronary risk factors, as well as with the increase of tertile risk factor among all patients at intermediate-risk profile. Categorizing CAD on the basis of stenosis severity alone fails to account for the continuum of risk associated with no obstructive atherosclerotic plaques [14]. It will be interesting to see whether the identification of vulnerable plaques by CTA will improve the risk stratification of future cardiac events, beyond the risk profile of patients at presentation [15]. In symptomatic patients with a suspicion of CAD, the recurrence of chest pain is frequent and usually patients undergo an additional functional test, due to the uncertainty of CAD presence, or undergo ICA directly, according to the behavior of the attending physician and the availability of local facilities and expertise. Thus, not surprisingly, in the USA [16], as well as in a European study [17], 40\% of ICA patients present no O-CAD, contributing to an inappropriate and unjustified consumption of financial resources. Currently, in patients with stable CAD, the rate of occurrence of events during a follow-up appears to be reduced, in comparison with that reported in Courage ERA [18] and, in a more recent study [3], it was as low as $1.5 \%$. Moreover, for symptomatic patients with normal coronary arteries, no additional evaluation test is necessary until 5 years from CTA according to the large CONFIRM study [19]. In our registry, no events were reported during the median 3-year followup for patients showing normal coronary arteries as well as no O-CAD. More recently, the 5-year clinical outcome of SCOT-HEART trial [20] showed that the use of CTA versus standard care alone is associated with a lower subsequent risk of death from CAD or nonfatal myocardial infarction (2.3\% vs. 3.9\%; HR 0.59). This benefit was achieved without a greater long-term use of ICA and coronary revascularization in the CTA group. According to the design of the study, the SCOT-HEART trial encouraged the secondary prevention strategy, i.e., statin agents, in patients with no O-CAD. This strategy may be very important, considering that nearly half of subsequent myocardial infarctions occurred among patients with no O-CAD. Finally, in patients showing OCAD involving small coronary vessels or marginal branches, we avoided performing ICA and angioplasty. These patients remained uneventful throughout the follow-up. In fact, in this setting, the advantage of angioplasty in comparison to optimal medical therapy remains to be demonstrated $[21,22]$. Thus, it is conceivable that, in the near future, the role of CTA as a prevention strategy could be expanded, thus avoiding 
invasive evaluation for patients with minimal or no O$\mathrm{CAD}$, as well as promoting preventive therapies, and finally, improving the appropriateness of ICA - avoiding coronary angioplasty in small vessels or minor branches showing lowrisk obstructive plaques.

4.1. Radiation Dose. The radiation dose remains the main concern regarding the use of CTA in clinical practice, due to the cancer risk it poses. According to ALARA dogma [11], all techniques should be improved in order to reduce the effective radiation dose. Our findings demonstrated a 6fold reduction of radiation dose by using high-pitch mode with respect to standard CTA mode. However, the feasibility of the high-pitch CTA mode depends on the patient heart rate. Despite the aggressive heart rate strategy control with beta-blocker/ivabradine adopted in our centre, the highpitch CTA mode was feasible in only half of the patients, underlying the difficulty in implementing the high-pitch CTA mode in clinical practice. It is important to note, however, that the radiation dose for nuclear stress testing was even higher, with an average level of $14 \mathrm{mSv}$, as noted in the literature, in comparison to that of the standard CTA scan [23]. In addition, the diagnostic certainty of chest pain may be lost, and the ICA examination may be necessary in the case of doubts raised by the results of functional tests. Finally, the lessening of the radiation dose obtained by this technique may affect the quality of the imaging. However, we note that in our registry, the reduction of effective radiation exposure obtained by high-pitch CTA mode did not have a significant impact on the imaging quality.

4.2. Limitations. This study was a single-center, observational registry with inherent limitations. First, due to the small sample size, caution is needed in the interpretation of the results. Second, the appropriateness of extrapolation of our findings to other centers will depend on the comparability of the clinical setting in terms of current diagnostic care, available technology, cost-accounting systems, and therapeutic management attitudes. Third, for the evaluation of outpatients with new onset chest pain, the combination of both anatomic and functional data could be the best diagnostic strategy [3]; however, the fractional flow reserve-CTA and the fusion/hybrid imaging is not available in our centre. Finally, in the present registry, the diagnostic CTA strategy is deemed to be cost-saving; however, the cost-effectiveness of CTA and the downstream health-care resource utilization both need to be evaluated in larger studies, though the NICE cost utility analysis of CTA appears favorable [7]. In addition, an important limitation of this registry is represented by the cost analysis performed by considering only the diagnostic workflow tests, whereas the cost saving related to the downstream ICA cancellation and avoided functional tests, and the additional costs related to the management of $\mathrm{O}$ CAD were not factored into the evaluation. Despite these limitations, we included outpatients who were representative of those referred to the cardiology clinic for assessment of suspected angina due to CAD. A similar rate of O-CAD was found in our chest pain population as in the larger SCOTHEART study $(17.8 \%$ vs. $24 \%)$, and a similar majority of our patients underwent an exercise test as those reported in the SCOT-HEART study (96\% vs. $85 \%$ ), confirming this overall picture. In our study, $42 \%$ of the exercise tests were inconclusive and $24 \%$ of patients with negative stress tests had obstructive CAD. Only $62.5 \%$ of positive stress tests had obstructive $\mathrm{CAD}$, confirming the moderate diagnostic value of the exercise test. Yet, considering the well-known overestimation of the prevalence of O-CAD based on the risk models [19] due to the low rate of O-CAD in real world populations with stable chest pain symptoms, CTA could be an efficient first-line test $[4,5,7]$. Thus, one should realize that in real-world setting, the use of CTA scan as a first-line diagnostic strategy for outpatients with new onset chest pain may be both feasible, safe, and cost-saving. However, a forward-looking economic investment strategy is necessary for the implementation and integration of CTA in the diagnostic workup for chest pain in the clinical practice [24].

4.3. Conclusions. Our registry confirms that in new onset chest pain patients no O-CAD was the pattern of atherosclerosis most commonly detected by the CTA, as first- or second-line investigation test. Implementation of CTA for clinical decision-making may influence the downstream diagnostic workflow of patients in real-world setting, leading us to safely avoid unnecessary ICA or additional functional tests. Although CTA appears to be cost-saving, the costeffectiveness of CTA integration in the diagnostic workflow for suspicion of CAD in real world remains to be demonstrated in larger studies.

\section{Data Availability}

The data used to support the findings of this study are available from the corresponding author upon request.

\section{Conflicts of Interest}

The authors declare no potential conflicts of interest with respect to the research, authorship, and/or publication of this article.

\section{Authors' Contributions}

All authors contributed to (1) the conception and design of the study, the acquisition, analysis, or interpretation of data for the work and gave final approval; (2) Vittorio Miele and Niccolò Marchionni critically revised the manuscript; and (3) Nazario Carrabba drafted the manuscript.

\section{References}

[1] J. A. Ladapo, S. Blecker, and P. S. Douglas, "Physician decision making and trends in the use of cardiac stress testing in the United States: An analysis of repeated cross-sectional data," Annals of Internal Medicine, vol. 161, no. 7, pp. 482-490, 2014. 
[2] G. Montalescot, U. Sechtem, S. Achenbach et al., "ESC guidelines on the management of stable coronary artery disease: the Task Force on the management of stable coronary artery disease of the European Society of Cardiology," European Heart Journal, vol. 34, no. 38, pp. 2949-3003, 2013.

[3] P. S. Douglas, B. De Bruyne, G. Pontone et al., "On behalf of the PLATFORM Investigators. 1-Year outcomes of FFRCT-Guided care in patients with suspected coronary disease: the PLATFORM study," Journal of the American College of Cardiology, vol. 68, pp. 435-445, 2016.

[4] P. S. Douglas, U. Hoffmann, M. R. Patel et al., "Outcomes of anatomical versus functional testing for coronary artery disease," The New England Journal of Medicine, vol. 372, pp. 1291-1300, 2015.

[5] Newby DE on behalf of the SCOT-HEART Investigators, "CT coronary angiography in patients with suspected angina due to coronary heart disease (SCOT-HEART): an open-label, parallel-group, multicentre trial," Lancet, vol. 385, no. 9985, pp. 2383-2391, 2015.

[6] D. Neglia, R. Daniele, C. Caselli et al., "Detection of significant coronary artery disease by noninvasive anatomical and functional imaging," Circulation: Cardiovascular Imaging, vol. 8, Article ID e002179, 2015.

[7] A. J. Moss, M. C. Williams, D. E. Newby, and E. D. Nicol, "The updated NICE guidelines: cardiac CT as the first-line test for coronary artery disease," Current Cardiovascular Imaging Reports, vol. 10, article no. 15, no. 5, 2017.

[8] J. Leipsic, S. Abbara, S. Achenbach et al., "SCCT guidelines for the interpretation and reporting of coronary ct angiography: a report of the society of cardiovascular computed tomography guidelines committee," Journal of Cardiovascular Computed Tomography, vol. 8, no. 5, pp. 342-358, 2014.

[9] Z. Wang, Y. Chen, Y. Wang et al., "Feasibility of low-dose contrast medium high pitch ct angiography for the combined evaluation of coronary, head and neck arteries," PLoS ONE, vol. 9, no. 3, p. e90268, 2014.

[10] W. G. Austen, J. E. Edwards, R. L. Frye et al., "A reporting system on patients evaluated for coronary artery disease. Report of the Ad Hoc Committee for Grading of Coronary Artery Disease, Council on Cardiovascular Surgery, American Heart Association," Circulation, vol. 51, supplement 4, pp. 5-40, 1975.

[11] J. Hausleiter, T. Meyer, F. Hermann et al., "Estimated radiation dose associated with cardiac CT angiography," Journal of the American Medical Association, vol. 301, no. 5, pp. 500-507, 2009.

[12] M. J. Budoff, D. Dowe, J. G. Jollis et al., "Diagnostic performance of 64-multidetector row coronary computed tomographic angiography for evaluation of coronary artery stenosis in individuals without known coronary artery disease: results from the prospective multicenter ACCURACY (Assessment by Coronary Computed Tomographic Angiography of Individuals Undergoing Invasive Coronary Angiography) trial," Journal of the American College of Cardiology, vol. 52, no. 21, pp. 1724-1732, 2008.

[13] B. J. Chow, G. Small, Y. Yam et al., "Prognostic and therapeutic implications of statin and aspirin therapy in individuals with nonobstructive coronary artery disease," Arteriosclerosis, Thrombosis, and Vascular Biology, vol. 35, no. 4, pp. 981-989, 2015.

[14] A. Arbab-Zadeh and V. Fuster, "The risk continuum of atherosclerosis and its implications for defining CHD by coronary angiography," Journal of the American College of Cardiology, vol. 68, no. 22, pp. 2467-2478, 2016.
[15] A. Ahmadi, J. Leipsic, R. Blankstein et al., "Do plaques rapidly progress prior to myocardial infarction?" Circulation Research, vol. 117, no. 1, pp. 99-104, 2015.

[16] M. R. Patel, E. D. Peterson, D. Dai et al., "Low diagnostic yield of elective coronary angiography," The New England Journal of Medicine, vol. 362, no. 10, pp. 886-895, 2010.

[17] L. Jespersen, A. Hvelplund, S. Z. Abildstrøm et al., "Stable angina pectoris with no obstructive coronary artery disease is associated with increased risks of major adverse cardiovascular events," European Heart Journal, vol. 33, no. 6, pp. 734-744, 2012.

[18] W. E. Boden, R. A. O'Rourke, K. K. Teo et al., "For the COURAGE Trial Research Group. Optimal medical therapy with or without PCI for stable coronary disease," The New England Journal of Medicine, pp. 1503-1516, 2007.

[19] B. J. Chow, G. Small, Y. Yam et al., "Incremental prognostic value of cardiac computed tomography in coronary artery disease using CONFIRM: Coronary Computed Tomography Angiography Evaluation for Clinical Outcomes: an International Multicenter Registry," Circulation: Cardiovascular Imaging, vol. 4, no. 5, pp. 463-472, 2011.

[20] The SCOT-HEART Investigators, "Coronary CT angiography and 5-year risk of myocardial infarction," The New England Journal of Medicine, vol. 379, pp. 924-933, 2018.

[21] R. Hachamovitch, S. W. Hayes, J. D. Friedman, I. Cohen, and D. S. Berman, "Comparison of the short-term survival benefit associated with revascularization compared with medical therapy in patients with no prior coronary artery disease undergoing stress myocardial perfusion single photon emission computed tomography," Circulation, vol. 107, no. 23, pp. 29002906, 2003.

[22] B. De Bruyne, W. F. Fearon, N. H. Pijls et al., "Fractional flow reserve-guided PCI for stable coronary artery disease," The New England Journal of Medicine, vol. 371, no. 13, pp. 1208-1217, 2014.

[23] T. C. Gerber, J. Jeffrey Carr, A. E. Arai et al., "Ionizing radiation in cardiac imaging: a science advisory from the American Heart Association Committee on cardiac imaging of the council on clinical cardiology and committee on cardiovascular imaging and intervention of the council on cardiovascular radiology and intervention," Circulation, vol. 119, no. 7, pp. 1056-1065, 2009.

[24] N. Carrabba, A. Migliorini, S. Pradella et al., "Old and new NICE guidelines for the evaluation of new onset stable chest pain: a real world perspective," BioMed Research International, vol. 2018, Article ID 3762305, 7 pages, 2018. 


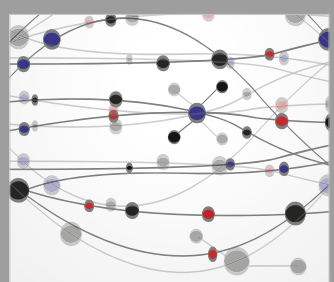

The Scientific World Journal
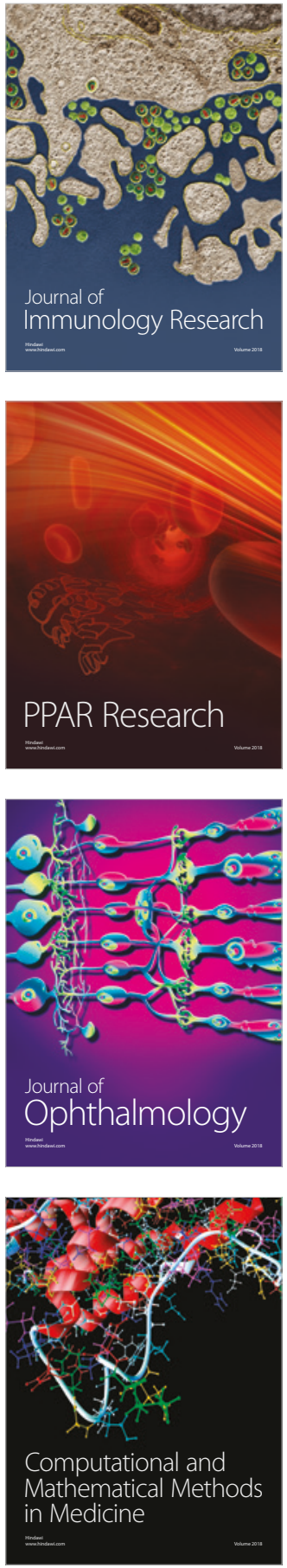

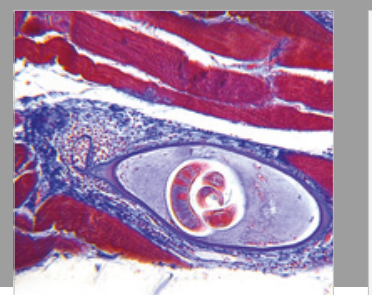

Gastroenterology Research and Practice

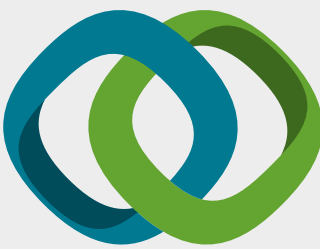

\section{Hindawi}

Submit your manuscripts at

www.hindawi.com
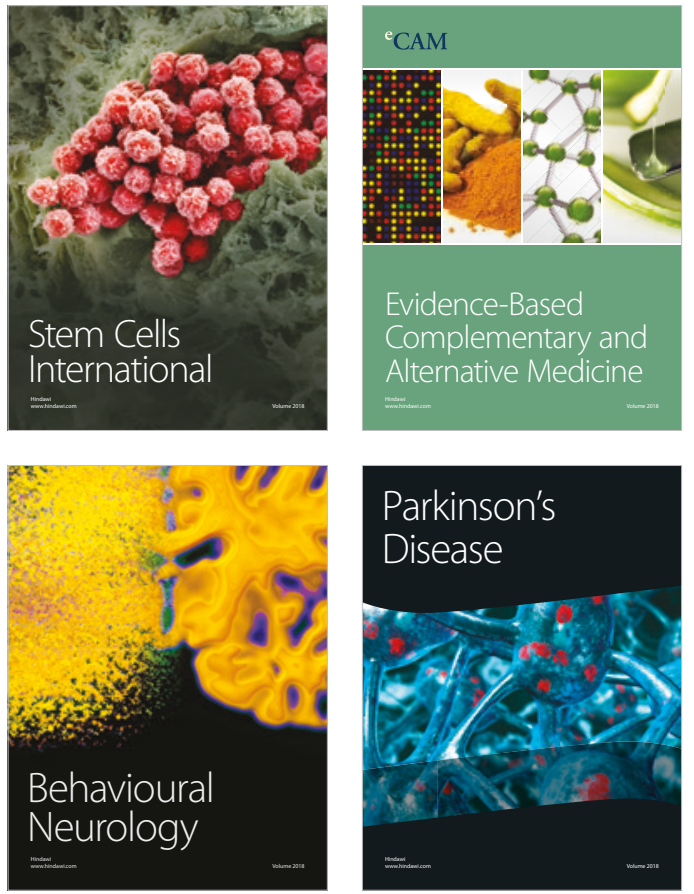

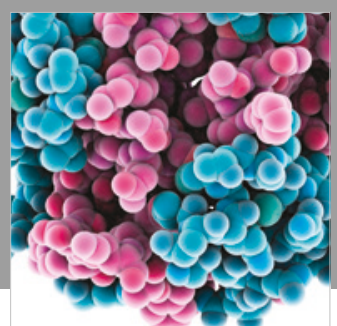

ournal of

Diabetes Research

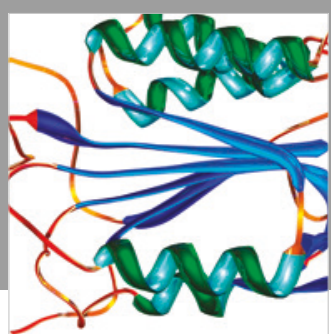

Disease Markers
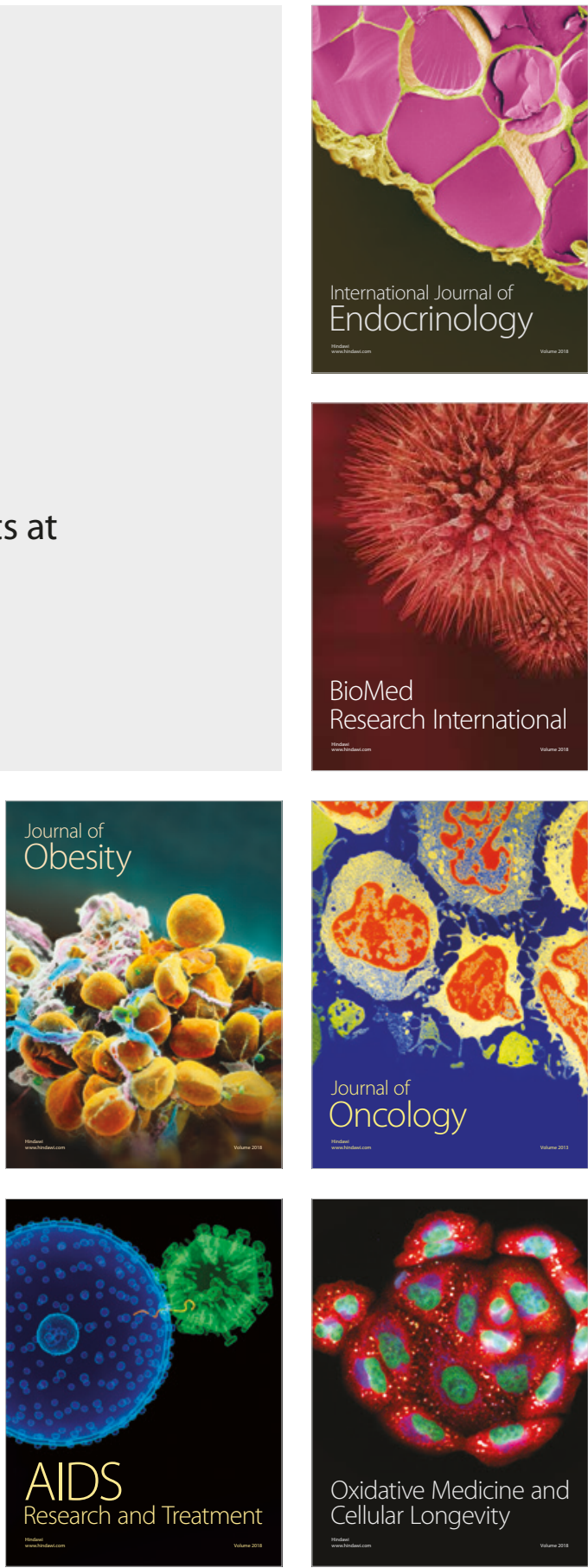